\title{
Adult hippocampal neurogenesis inversely correlates with microglia in conditions of voluntary running and aging
}

\author{
Elias Gebara, Sebastien Sultan, Jacqueline Kocher-Braissant and Nicolas Toni*
}

Department of Fundamental Neurosciences, University of Lausanne, Lausanne, Switzerland

\section{Edited by:}

Jack M. Parent, University of

Michigan, USA

Reviewed by:

Djoher N. Abrous, Institut des

Neurosciences de Bordeaux, France

Amanda Sierra, University of the

Basque Country EHU/UPV, Spain

\section{${ }^{*}$ Correspondence:}

Nicolas Toni, Department of

Fundamental Neurosciences,

University of Lausanne, 9, rue du

Bugnon, 1005 Lausanne,

Switzerland

e-mail:nicolas.toni@unil.ch
Adult hippocampal neurogenesis results in the formation of new neurons and is a process of brain plasticity involved in learning and memory. The proliferation of adult neural stem or progenitor cells is regulated by several extrinsic factors such as experience, disease or aging and intrinsic factors originating from the neurogenic niche. Microglia is very abundant in the dentate gyrus (DG) and increasing evidence indicates that these cells mediate the inflammation-induced reduction in neurogenesis. However, the role of microglia in neurogenesis in physiological conditions remains poorly understood. In this study, we monitored microglia and the proliferation of adult hippocampal stem/progenitor cells in physiological conditions known to increase or decrease adult neurogenesis, voluntary running and aging respectively. We found that the number of microglia in the DG was strongly inversely correlated with the number of stem/progenitor cells and cell proliferation in the granule cell layer. Accordingly, co-cultures of decreasing neural progenitor/glia ratio showed that microglia but not astroglia reduced the number of progenitor cells. Together, these results suggest that microglia inhibits the proliferation of neural stem/progenitor cells despite the absence of inflammatory stimulus.

Keywords: adult neurogenesis, dentate gyrus, microglia, running, aging

\section{INTRODUCTION}

In the mammalian brain, adult neural stem cells reside in the subventricular zone and subgranular zone (SGZ) of the hippocampus and continue to produce new neurons throughout life (Altman, 1969). Adult neurogenesis has been observed in all mammalian species including humans (Eriksson et al., 1998) and results in the formation of new neurons in the olfactory bulb and the dentate gyrus (DG) of the hippocampus. Radial glia-like (RGL) neural stem cells that reside in the SGZ of the DG, proliferate and give rise to transit-amplifying progenitors (TAP) expressing the T-box brain gene 2 (Tbr2) antigen which then give rise to doublecortin (DCX)-expressing immature neurons (Gage, 2000; Yao et al., 2012). After a phase of maturation, newly-formed neurons express the mature neuronal marker $\mathrm{Neu} \mathrm{N}$, functionally integrate into the hippocampal network (Toni and Sultan, 2011) and participate to mechanisms of learning and memory (Aimone et al., 2010). Each of these steps is highly regulated by signaling from the neurogenic niche, and an increasing number of pro-neurogenic factors are being discovered, with great potential interest for cell-replacement therapeutic approaches. The neurogenic niche is constituted by stem cells and their progenies, astrocytes, oligodendrocytes, endothelial cells, microglia, mature and immature neurons (Shihabuddin et al., 2000; Song et al., 2002; Zhao et al., 2008; Bonaguidi et al., 2011). These cells release a wide array of factors that control adult neurogenesis. However, the contribution of specific cell types on the processes of adult neurogenesis remains poorly determined.

Of particular interest, microglia are abundant and widely distributed throughout the adult brain. These cells are involved in the inflammatory reaction and act as the resident immune cells of the brain. Their fine extensions and dynamic nature enable microglia to survey the entire brain parenchyma for potential infection or cell damage and, upon activation, they release cytokines and proceed to the phagocytosis of cell debris or infectious micro-organisms. The experimental activation of microglia by the administration of the bacterial endotoxin lipopolysaccharide (LPS) has been shown to decrease adult neurogenesis, by specifically inhibiting the proliferation or the survival of the new cells (Ekdahl et al., 2003; Monje et al., 2003; Fujioka and Akema, 2010). These effects may be mediated by the release of cytokines such as IL- $6, \mathrm{TNF} \alpha$, IL-1 $1 \beta$, since these molecules show an inhibitory effect on adult neurogenesis in vitro or in vivo (Vallieres et al., 2002; Monje et al., 2003; Keohane et al., 2010; Kohman and Rhodes, 2013).

Recently, the role of microglia in absence of lesion or inflammation has started to raise interest. Indeed, recent studies suggest that resting microglia may be involved in the regulation of physiological mechanisms such as dendritic spine maintenance (Paolicelli et al., 2011) or the homeostasis of the neurogenic niche by the removal of apoptotic newborn cells (Sierra et al., 2010). Furthermore, neural progenitor cells (NPCs) regulate microglia function in vitro, by the secretion of growth factors (Mosher et al., 2012). However, the role of microglia on adult neurogenesis in physiological conditions remains unclear.

Here, we examined the correlation between the amount of microglia in the hippocampus and the proliferation of adult neural stem cells, in physiological conditions. To increase the variability in progenitor proliferation, we used aging and voluntary 
exercise, as these parameters are known to decrease and increase neurogenesis respectively (Kuhn et al., 1996; van Praag et al., 1999, 2005; Encinas et al., 2011; Kempermann, 2011).

\section{MATERIALS AND METHODS ETHICS STATEMENT}

This study was carried out in strict accordance with the recommendations in the Guidance for the Care and Use of Laboratory Animals of the National Institutes of Health. All experimental protocols were approved by the Swiss animal experimentation authorities (Service de la consommation et des affaires vétérinaires, Chemin des Boveresses 155, 1066 Epalinges, Switzerland, permit number: 2301). Every effort was made to minimize the number of animals used and their suffering.

\section{EXPERIMENTAL ANIMALS}

All animals used for this study were adult male mice. GFAP-GFP mice were a kind gift from the laboratory of Helmut Kettenmann (Max-Delbruck center, Berlin, Germany) (Nolte et al., 2001). They express the green fluorescent protein (GFP) under the control of the human glial fibrillary acidic protein promoter (GFAP). At the beginning of the experiment, the first group of mice was 6-week-old and the second group 7.5-month-old. Runner mice were housed for 2 weeks in standard cages equipped with a running wheel (Fast-Trac; Bio-Serv, USA) and were allowed free access to the running wheel. Non-runner mice were housed in similar, adjacent cages without running wheel. All mice were housed in a 12-h light/dark cycle and controlled temperature of $22^{\circ} \mathrm{C}$. Food and water were available ad-libitum.

\section{BrdU ADMINISTRATION}

Immediately at the end of the running period, all mice were injected intraperitoneally with the DNA replication marker, Bromodeoxyuridine (BrdU, Sigma-Aldrich, Buchs, Switzerland), at doses of $100 \mathrm{mg} / \mathrm{kg}$ in saline, 3 times at 2-h intervals. Two hours after the last injection, mice were sacrificed and the number of BrdU cell was counted to assess cell proliferation (Mandyam et al., 2007; Taupin, 2007; Yang et al., 2011; Gao and Chen, 2013).

\section{TISSUE COLLECTION AND PREPARATION}

At the end of the experiment, mice received a lethal dose of pentobarbital (10 mL/kg, Sigma-Aldrich, Buchs, Switzerland) and were perfusion-fixed with $50 \mathrm{ml}$ of $0.9 \%$ saline followed by $100 \mathrm{~mL}$ of $4 \%$ paraformaldehyde (Sigma-Aldrich, Switzerland) dissolved in phosphate buffer saline (PBS 0.1M, pH 7.4). Brains were then collected, postfixed overnight at $4^{\circ} \mathrm{C}$, cryoprotected $24 \mathrm{~h}$ in $30 \%$ sucrose and rapidly frozen. Coronal frozen sections of a thickness of $40 \mu \mathrm{m}$ were cut with a microtome-cryostat (Leica MC $3050 S$ ) and slices were kept in cryoprotectant (30\% ethylene glycol and $25 \%$ glycerin in $1 \mathrm{X}$ PBS) at $-20^{\circ} \mathrm{C}$ until processed for immunostaining.

\section{IMMUNOHISTOCHEMISTRY}

Immunochemistry was performed on 1-in-6 series of section. Sections were washed 3 times in PBS $0.1 \mathrm{M}$. BrdU detection required formic acid pretreatment (formamide $50 \%$ in $2 \mathrm{X}$ SSC buffer; $2 \mathrm{X} \mathrm{SSC}$ is $0.3 \mathrm{M} \mathrm{NaCl}$ and $0.03 \mathrm{M}$ sodium citrate, $\mathrm{pH} 7.0$ ) at $65^{\circ} \mathrm{C}$ for $2 \mathrm{~h}$ followed by DNA denaturation for $30 \mathrm{~min}$ in $2 \mathrm{M} \mathrm{HCl}$ for $30 \mathrm{~min}$ at $37^{\circ} \mathrm{C}$ and rinsed in $0.1 \mathrm{M}$ borate buffer $\mathrm{pH} 8.5$ for $10 \mathrm{~min}$. Then, slices were incubated in blocking solution containing $0.3 \%$ Triton-X100 and $15 \%$ normal serum normal goat serum (Gibco, 16210-064) or normal donkey serum (Sigma Aldrich, D-9663), depending on the secondary antibody in PBS $0.1 \mathrm{M}$. Slices were then incubated $40 \mathrm{~h}$ at $4^{\circ} \mathrm{C}$ with the following primary antibodies: mouse monoclonal anti-BrdU (1:250, Chemicon International, Dietikon, Switzerland), rabbit anti-Ki-67 (1:200, Abcam, ab15580), goat anti-DCX (1:500, Santa Cruz biotechnology, sc-8066), rabbit anti-GFAP (1:500, Invitrogen, 180063), rabbit anti-Tbr2 (1:200, Abcam, ab23345), goat anti-Iba1 (1:200, Abcam, ab5076), mouse anti-MHC-II (1:200 Abcam, ab23990). The sections were then incubated for $2 \mathrm{~h}$ in either of the following secondary antibodies: goat anti-mouse Alexa-594 (1:250, Invitrogen), goat anti-rabbit Alexa-594 (1:250, Invitrogen), donkey anti-goat Alexa-555 (1:250, Invitrogen). 4,6 diamidino-2-phenylindole (Dapi) was used to reveal nuclei.

\section{CELL CULTURE}

Adult NPCs expressing the red fluorescent protein (RFP) are a kind gift from the laboratory of Fred Gage (Salk Institute, San Diego, USA). They were isolated from the DG of adult Fisher 344 rats and cultured as previously described (Palmer et al., 1997). Microglia and astrocyte primary culture were purified from postnatal day 3 rats. Cerebral cortices, including the hippocampus, were mechanically triturated for homogenization and seeded onto poly-D-lysine coated $75 \mathrm{~cm}^{2}$ flasks in Dulbecco's Modified Eagle Medium (DMEM) glutamax (Invitrogen, USA), 10\% normal calf serum with penicillin/streptomycin (Invitrogen, USA). Cells were grown for 5-7 days in a humidified 5\% CO2 incubator at $37^{\circ} \mathrm{C}$. At confluence, flasks were shaken at $250 \mathrm{rpm}$ on an orbital shaker for $2 \mathrm{~h}$ to separate microglia from astrocytes. Detached microglia were seeded in poly-D-lysine coated 6-well microplates in culture medium supplemented with $30 \%$ astrocyte conditioned medium, to enhance the survival of microglia (personal communication from Dr. Romain Gosselin, University of Lausanne). Different numbers of microglia or astrocytes were plated on coated $12 \mathrm{~mm}$ coverslips in a 24-well culture plate $(20,000,40,000,60,000$ cells per well). Three hours later, a fixed number of RFP-expressing NPCs (20,000 cells per well) were plated on the same culture wells, at a (NPCs/Glia) ratio of $1 / 0$, $1 / 1,1 / 2,1 / 3$. Three wells per condition were used. Ninety-six hours after plating, cells were fixed with $4 \%$ paraformaldehyde for $20 \mathrm{~min}$, briefly washed and immunostained for Ibal or GFAP and mounted.

\section{CELL NUMBER QUANTIFICATION}

All images were acquired using a confocal microscope (Zeiss LSM 710 Quasar Carl Zeiss, Oberkochen, Germany). The total number of immunoreactive cells was estimated throughout the entire granule cell layer using stereological sampling, as previously described (Thuret et al., 2009), between -1.3 to $-2.9 \mathrm{~mm}$ from the Bregma. For each animal, a 1-in-6 series of sections was stained with the nucleus marker DAPI and used to measure the volume of the granule cell layer. The granule cell area was traced using Axiovision (Zeiss, Germany) software and the 
granule cell volume was determined by multiplying the traced granule cell layer area by the thickness of the corresponding section and the distance between the sections sampled $(240 \mu \mathrm{m})$. For all mice analyzed in this study, no difference of volume was found between groups [One-Way ANOVA, $F_{(3,15)}=0.29, p=0.82$ ], (data not shown). All cells were counted blind with regard to the mouse status. The number of labeled cells was counted for each section, in the entire thickness of the granular cell layer of the DG with a 40x objective. RGL cells and cells expressing BrdU, Ki-67, DCX or Tbr2 were counted in an area containing the granule cell layer and the subgranular zone, whereas cells expressing Iba1, MHC-II and GFAP (Figures 2A,D,F) were counted in an area including the sub-granular cell layer, the granule cell layer, the molecular layer and the hilus. Cells expressing Ibal (Figure 2B) were also counted in the primary somatosensory cortex (S1).

For in vitro cell quantification, images were acquired using confocal microscopy. The numbers of RFP-expressing NPCs, Iba1-expressing microglia and GFAP-expressing astrocytes were counted in 4 selected fields, systematically placed in the same positions relative to the coverslips' edges. The total number of cells per selected field was divided by the total area of the selected fields to obtain an average cell density per well that was then multiplied by the total surface area of the coverslip to obtain an estimate of the total number of cells per coverslip. This number of cells was then compared to the number of cells that were plated in the wells to obtain a percentage of increase in NPCs number.

\section{STATISTICAL ANALYSIS}

Hypothesis testing was two-tailed. All analyses were performed using JMP10 software. First, Shapiro-Wilk tests were performed on each dataset to test for distribution normality. The distribution was normal for all data. To test for possible interaction between the 2 groups (Aging and Running) a Two-Way ANOVA was performed. When an interaction was found, a One-Way ANOVA test was performed, if no interaction was found, we analyzed each variable independently using a Two-Way ANOVA. All analyses were followed by a post-hoc Tukey HSD test. In order to test linear correlation, Spearman correlation test was performed. Results are expressed by the Spearman correlation coefficient $(\rho)$ and the $p$-value. Data is presented as mean \pm standard error of the mean (SEM).

\section{RESULTS}

\section{EFFECT OF AGING AND RUNNING ON CELL PROLIFERATION AND IMMATURE NEURONS}

We tested the effect of running and aging on GFAP-GFP mice (Nolte et al., 2001), which are commonly-used mice models for the examination of adult neurogenesis and enable the identification of stem cells (Huttmann et al., 2003; Ehninger and Kempermann, 2008). Sixteen GFAP-GFP mice were divided in 4 experimental groups: young adult mice ( 8 weeks of age; $8 \mathrm{~W}$ ) and older mice ( 8 months of age; $8 \mathrm{M}$ ), that were previously housed individually in standard cages in presence (R) or absence (NR) of a running wheel for 2 weeks ( 4 mice per group). At the end of the 2 weeks period, all mice received 3 intraperitoneal injections of BrdU $(100 \mathrm{mg} / \mathrm{kg})$ at $2 \mathrm{~h}$ intervals and were sacrificed $2 \mathrm{~h}$ after the last BrdU injection (Figure 1A). Brains were sectioned and immunostained for BrdU and markers for adult neurogenesis and microglia. We first assessed cell proliferation in the granule cell layer of the DG. A Two-Way ANOVA test revealed that there is interaction between aging and running for Ki-67 marker $\left[F_{(1,12)}=5.74 ; p<0.05\right]$. The density of Ki-67-expressing cells was significantly decreased by aging [Figures 1B,C, One-Way ANOVA $F_{(3,15)}=22.65 ; p<0.001$. Post-hoc Tukey HSD test: NR $8 \mathrm{~W}$ vs. NR $8 \dot{M} p<0.05$ ] and increased by running [One-Way ANOVA $F_{(3,15)}=22.65 ; p<0.001$. Post-hoc Tukey HSD test NR $8 \mathrm{~W}$ vs. R $8 \mathrm{~W} p<0.001$, NR $8 \mathrm{M}$ vs. $\mathrm{R} 8 \mathrm{M} p=0.24]$.

Similarly, there is interaction between aging and running for BrdU marker [Two-Way ANOVA $F_{(1,12)}=17.6 ; p<0.01$ ]. The density of BrdU-expressing cells was significantly decreased with aging [Figures 1D,E, One-Way ANOVA $F_{(3,15)}=62.19$; $p<0.001$. Post-hoc Tukey HSD test: NR $8 \mathrm{~W}$ vs. NR $8 \mathrm{M}$, $p<0.05$ ] and increased by running [One-Way ANOVA $F_{(3,15)}=$ 62.19; $p<0.001$. Post-hoc Tukey HSD test: NR $8 \mathrm{~W}$ vs. R $8 \mathrm{~W}$ $p<0.001$, NR $8 \mathrm{M}$ vs. R $8 \mathrm{M} p<0.01]$. Thus, cell proliferation was increased by voluntary running and reduced by aging.

In the DG, proliferative cells are divided in 2 main cell populations: the type 1, radial glia-like (RGL) stem cells and the type 2, TAPs. RGL cells were identified by the expression of GFP, their nucleus located in the subgranular zone and a radial process extending through the granule cell layer and branching into the molecular layer (Huttmann et al., 2003; Mignone et al., 2004; Kriegstein and Alvarez-Buylla, 2009; Beckervordersandforth et al., 2010). They expressed the selfrenewal factor Sox2 (data not shown). For RGL, Two-Way ANOVA test showed that there is interaction between aging and running $\left[F_{(1,12)}=5.3 ; p<0.05\right]$. The density of RGL cells was significantly decreased by aging (Figures 1F,G, One-Way ANOVA $F_{(3,15)}=35.81 ; p<0.001$. Post-hoc Tukey HSD test: NR $8 \mathrm{~W}$ vs. NR $8 \mathrm{M} p<0.05$ ] and increased by running [One-Way ANOVA $F_{(3,15)}=35.81 ; p<0.001$. Post-hoc Tukey HSD test: NR $8 \mathrm{~W}$ vs. $\mathrm{R} 8 \mathrm{~W} p<0.001$, NR $8 \mathrm{M}$ vs. $\mathrm{R} 8 \mathrm{M} p<0.01$ ]. TAPs were identified by immunostaining against Tbr2 (Hodge et al., 2008). Similarly, there is interaction between aging and running for Tbr2 marker [Two-Way ANOVA $F_{(1,12)}=5.2 p<0.05$ ]. The density of Tbr2-expressing cells was decreased by aging [Figures 1H,I, OneWay ANOVA $F_{(3,15)}=27.32 ; p<0.001$. Post-hoc Tukey HSD test: NR $8 \mathrm{~W}$ vs. NR $8 \mathrm{M} p<0.05$ ] and increased by running [One-Way ANOVA $F_{(3,15)}=27.32 ; p<0.001$. Post-hoc Tukey HSD test NR $8 \mathrm{~W}$ vs. R $8 \mathrm{~W} p<0.001$, NR $8 \mathrm{M}$ vs. $\mathrm{R} 8 \mathrm{M} p=0.2$ ]. Thus, running increased and aging decreased the density of stem and progenitor cells.

Finally, we examined the effect of aging and running on immature neurons identified by immunohistochemistry against doublecortin (DCX). A Two-Way ANOVA test revealed that there is no interaction between aging and running for DCX marker $\left[F_{(1,12)}=2.21 ; p=0.16\right]$. Although, The density of DCX-expressing cells decreased with aging [Figures 1J,K, Anova $F_{(1,12)}=52.90 ; p<0.001$. Post-hoc Tukey HSD test: NR $8 \mathrm{~W}$ vs. NR 8 M $p<0.01]$ and increased with running $\left[F_{(1,12)}=29.97\right.$; $p<0.001$. Post-hoc Tukey HSD test NR $8 \mathrm{~W}$ vs. R $8 \mathrm{~W} p<0.001$, 

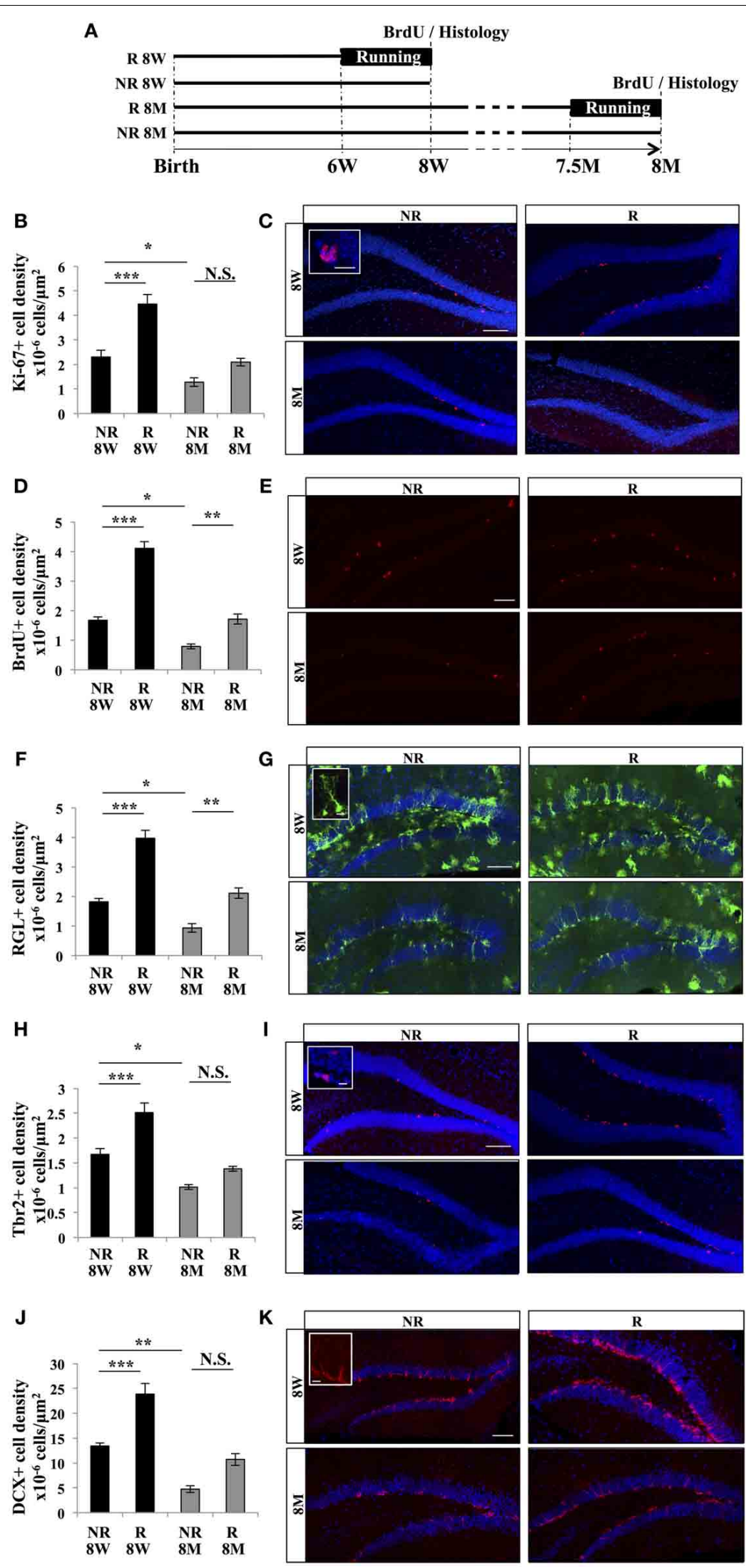

FIGURE 1 | Continued 
FIGURE 1 | Effect of running and aging on cell proliferation in the dentate gyrus. (A) Experimental timeline. (B) Histogram of the density (cells $/ \mu \mathrm{m}^{2}$ ) of Ki-67-expressing cells in the granule cell layer of the dentate gyrus of 8-week-old animals $(8 \mathrm{~W})$ and 8 -month-old animals (8M) housed with (R), or without (NR) a running wheel. (C) Confocal maximal projection micrographs of hippocampal sections immunostained for Ki-67. Inset: Higher magnification confocal micrograph of a Ki-67-expressing cell. (D) Histogram of the density of BrdU-positive cells in the granule cell layer of the dentate gyrus. (E) Confocal maximal projection micrographs of hippocampal sections immunostained for BrdU. (F) Quantification of the density of RGL cells in the granule cell layer of the dentate gyrus. (G) Confocal maximal projection micrographs of hippocampal sections.
Inset: Higher magnification confocal micrograph of a RGL cell. (H) Histogram showing the density of Tbr2-expressing cells in the granule cell layer of the dentate gyrus. (I) Confocal maximal projection micrographs of hippocampal sections immunostained for Tbr2. Inset: Higher magnification confocal micrograph of a Tbr2-expressing cell. (J) Histogram of the density of DCX-immunolabeled cells in the granule cell layer of the dentate gyrus. (K) Confocal micrographs of hippocampal sections immunostained for DCX. Inset: Higher magnification confocal micrograph of a DCX-immunolabeled group of cells. Blue: Dapi staining. Animals, $n=4$ per group. Scale bars: $100 \mu \mathrm{m}$, insets $10 \mu \mathrm{m}$, post-hoc Tukey HSD test ${ }^{*} p<0.05 ;{ }^{* *} p<0.01 ;{ }^{* *} p<0.001$; NS: $p>0.05$. Each value represents the mean \pm SEM.
NR $8 \mathrm{M}$ vs. $\mathrm{R} 8 \mathrm{M} p=0.06]$. Together, these results indicate that the density of proliferative cells, cell proliferation and the density of immature neurons decreased with aging and increased with voluntary running.

\section{EFFECT OF AGING AND RUNNING ON MICROGLIA}

Next we examined the effect of aging and running on microglia, using the immunomarker Iba1. Iba1-expressing cells displayed an oval cell body and numerous ramified processes (Figures $2 \mathrm{~A}-\mathrm{C}$ ). A Two-Way ANOVA revealed that there is interaction between aging and running for Iba1 marker $\left[F_{(1,12)}=5.65 ; p<0.05\right]$. The density of Iba 1-expressing cells in the DG was decreased by running [Figures $2 \mathrm{~A}-\mathrm{C}$, One-Way ANOVA $F_{(3,15)}=75.94$; $p<0.001$. Post-hoc Tukey HSD test: NR 8 W vs. R 8 W $p<0.001$, NR $8 \mathrm{M}$ vs. R $8 \mathrm{M} p<0.001$ ] and increased by aging [One-Way ANOVA $F_{(3,15)}=75.94 ; p<0.001$. Post-hoc Tukey HSD test: NR $8 \mathrm{~W}$ vs. NR $8 \mathrm{M} p<0.001]$. To examine whether the effect of running and aging on microglia was restricted to the hippocampus, we measured microglia in the primary somato-sensory cortex of all mice. Contrary to what we observed in the DG, in the primary somato-sensory cortex, there was no interaction between aging and running in the level of Ibal marker [Two-Way ANOVA $\left.F_{(1,12)}=2.1 ; p=0.17\right]$. Anova showed that running increased [Figure 2B, $F_{(1,12)}=92.47 ; p<0.001$. Post-hoc Tukey HSD test: NR $8 \mathrm{~W}$ vs. R $8 \mathrm{~W} p<0.001$, NR $8 \mathrm{M}$ vs. R $p<0.001]$ whereas aging decreased the density of microglia in the cortex $\left[F_{(1,12)}=\right.$ 52.49; $p<0.001$. Post-hoc Tukey HSD test: NR $8 \mathrm{~W}$ vs. NR $8 \mathrm{M}$ $p<0.001]$.

To test whether the expression of the antigen presentation protein MHC II was also regulated by aging and running, we immunostained brain slices for MHC II. A Two-Way ANOVA revealed that there is interaction between aging and running for MHC II marker $\left[F_{(1,12)}=8.08 p<0.01\right]$. The density of MHC II-expressing cells was decreased by running [Figures 2D,E, OneWay ANOVA $F_{(3,15)}=145.19 ; p<0.001$. Post-hoc Tukey HSD test: NR $8 \mathrm{~W}$ vs. R $8 \mathrm{~W} p<0.001$, NR $8 \mathrm{M}$ vs. R $8 \mathrm{M} p<0.001]$ and increased by aging [One-Way ANOVA $F_{(3,15)}=145.19, p<$ 0.001. Post-hoc Tukey HSD test: NR $8 \mathrm{~W}$ vs. NR $8 \mathrm{M} p<0.001]$. Finally, we examined whether astroglia was similarly affected by running or aging by immunostaining against GFAP. The density of GFAP-expressing astrocytes was constant throughout all conditions [Figures 2F,G; Two-Way ANOVA $F_{(3,12)}=0.49, p=0.69$ ], indicating that the effect of aging and running was specific to microglia.

\section{CORRELATION BETWEEN MICROGLIA AND ADULT NEUROGENESIS}

The opposite effects of running and aging on microglia and adult neurogenesis suggest that microglia and adult neurogenesis may be inversely correlated. We therefore plotted, for each mouse, the number of Iba-expressing microglia and the number of cells expressing markers for neurogenesis. The number of Iba1expressing microglia was inversely correlated with the number of Ki-67-expressing cells (Figure 3; $\rho=-0.92, p<0.001$ ), RGL cells $(\rho=-0.98, p<0.001)$, Tbr2-expressing cells $(\rho=-0.85$, $p<0.001)$, BrdU-immunolabeled cells $(\rho=-0.95, p<0.001)$, and DCX-expressing cells $(\rho=-0.88, p<0.001)$. Similarly, the number of $\mathrm{MCH}$ II-expressing microglia inversely correlated with the number of Ki-67-expressing cells (Data not shown; $\rho=-0.95, p<0.001)$, RGL cells $(\rho=-0.92, p<0.001)$, Tbr2expressing cells $(\rho=-0.97, p<0.001)$, BrdU-immunolabeled cells $(\rho=-0.94, \quad p<0.001)$, and DCX-expressing cells $(\rho=-0.97, p<0.001)$. These results indicate that microglia density was inversely correlated with stem/progenitor cell proliferation.

To test the possibility that microglia number could directly affect stem/progenitor cell proliferation, we co-cultured microglia and NPCs. We plated a constant number of NPCs with an increasing proportion of microglia and 4 days later, we counted the number of remaining NPCs. The number of NPCs significantly decreased with the increasing proportion of co-cultured microglia [Figure 4; One-Way ANOVA $F_{(6,20)}=82.58, p<0.001 ; 1 / 0$ vs. $1 / 1$ post-hoc Student's $t$-test $p<0.001,1 / 0$ vs. $1 / 2$ post-hoc Student's $t$-test $p<0.001,1 / 0$ vs. $1 / 3$ post-hoc Student's $t$-test $p<0.001]$. To test whether this effect was specific to microglia, we repeated this experiment with increasing proportion of astrocytes instead of microglia. In contrast to microglia, astrocytes did not decrease the number of NPCs in co-cultures [One-Way ANOVA $F_{(6,20)}=82.58, p<0.001 ; 1 / 0$ vs. $1 / 2$ post-hoc Student's $t$-test $p=0.53,1 / 0$ vs. $1 / 3$ post-hoc Student's $t$-test $p=0.13$ ]. Thus, increasing microglia density inhibited NPCs growth and/or survival in vitro and resulted in a corresponding decrease in NPCs number.

\section{DISCUSSION}

In this study, we exploited the physiological variations of adult neurogenesis induced by voluntary running and aging to examine the correlation between microglia and adult hippocampal neurogenesis in absence of inflammatory stimulus. In line with previous studies, we found that aging decreased cell proliferation and the 

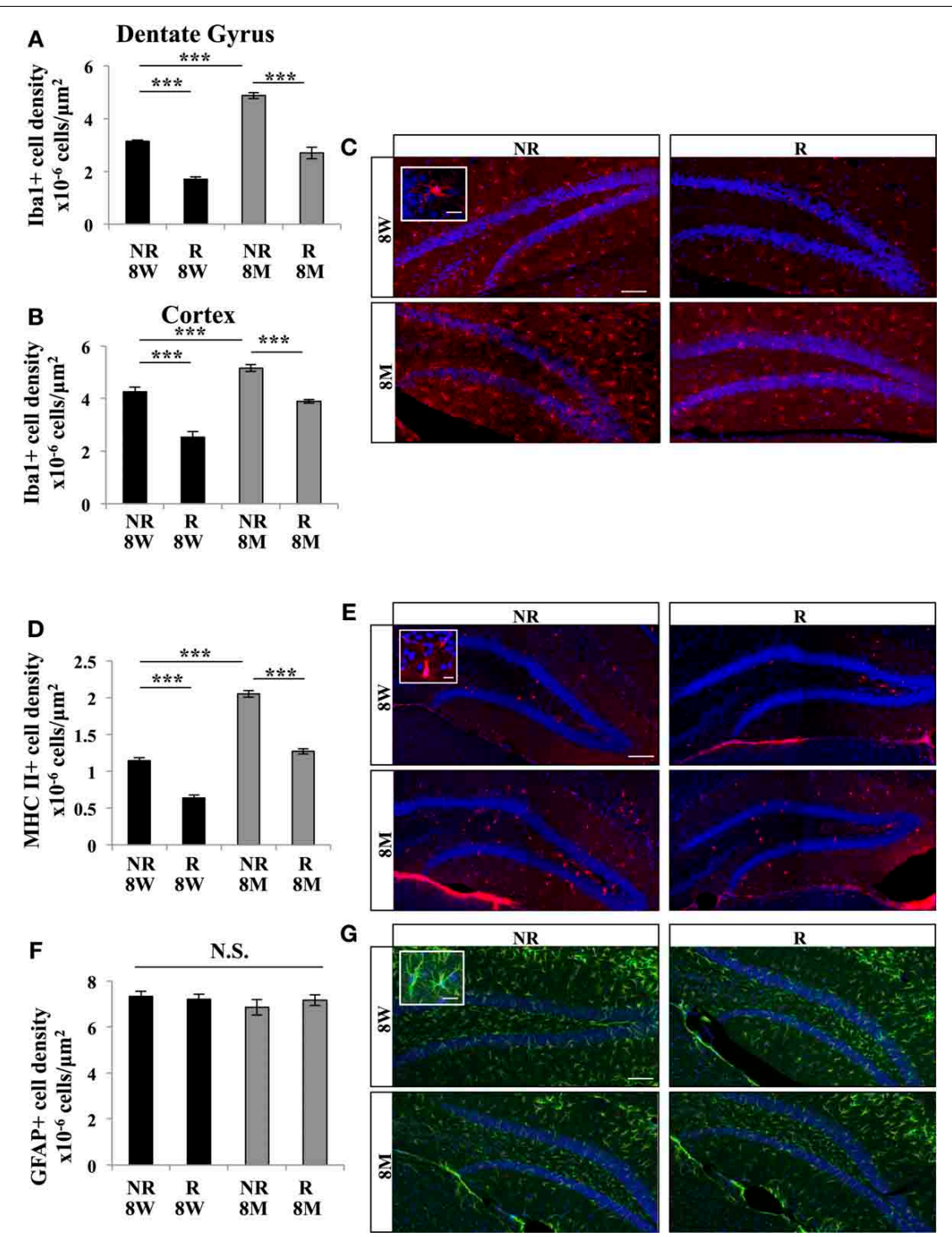

FIGURE 2 | Effect of running and aging on the density of microglia. (A) Histogram showing the density (cells $/ \mu \mathrm{m}^{2}$ ) of lba1-expressing cells in the dentate gyrus (including hilus, granule cell layer and molecular layer). (B) Histogram of the density of Iba1-expressing cells in the cortex. (C) Confocal maximal projection micrographs of hippocampal sections immunostained for lba1. Inset: Higher magnification confocal micrograph of an Iba1-immunolabeled cell. (D) Histogram of the density of $\mathrm{MHC}$ II-expressing cells in the dentate gyrus.

(E) Confocal maximal projection micrographs of hippocampal sections immunostained for MHC II. Inset: Higher magnification confocal micrograph of a MHC II-immunolabeled cell. (F) Histogram of the density of GFAP-expressing astrocytes in the dentate gyrus of mice for each experimental condition. (G) Confocal maximal projection micrographs of hippocampal sections immunostained for GFAP. Inset: Higher magnification confocal micrograph of a GFAP-immunolabeled cell. Blue: Dapi staining. Animals: $n=4$ per group. Scale bars: $100 \mu \mathrm{m}$, insets $10 \mu \mathrm{m}$. post-hoc Tukey HSD test: N.S.: $p>0.05$; ${ }^{* * *} p<0.001$. Each value represents the mean \pm SEM. number of immature neurons whereas voluntary running had inverse effects (Kuhn et al., 1996; van Praag et al., 1999, 2005; Encinas et al., 2011; Kempermann, 2011). In addition, we found that the decrease in RGL cells observed with aging (Encinas et al., 2011), was reversed by voluntary running, suggesting that running may induce the symmetrical division of RGL cells and restore their population. Strikingly, in both conditions, the amount of microglia but not of astroglia was strongly inversely correlated with all measured parameters of neurogenesis. Similarly, in vitro, co-cultures of NPCs with an increasing proportion of microglia but not of astroglia reduced the number of NPCs after 4 days. Together, these results indicate that, in physiological conditions, microglia and neurogenesis are inversely correlated and suggest that microglia may inhibit adult neurogenesis by directly acting on stem/progenitor cells.

These observations are in line with previous experiments showing that, in inflammatory conditions induced by epilepsy, ischemia or LPS injection, microglia undergo dramatic changes in their morphological and cytokine expression pattern and inhibit adult neurogenesis (Monje et al., 2002; Ekdahl et al., 2003; Liu 

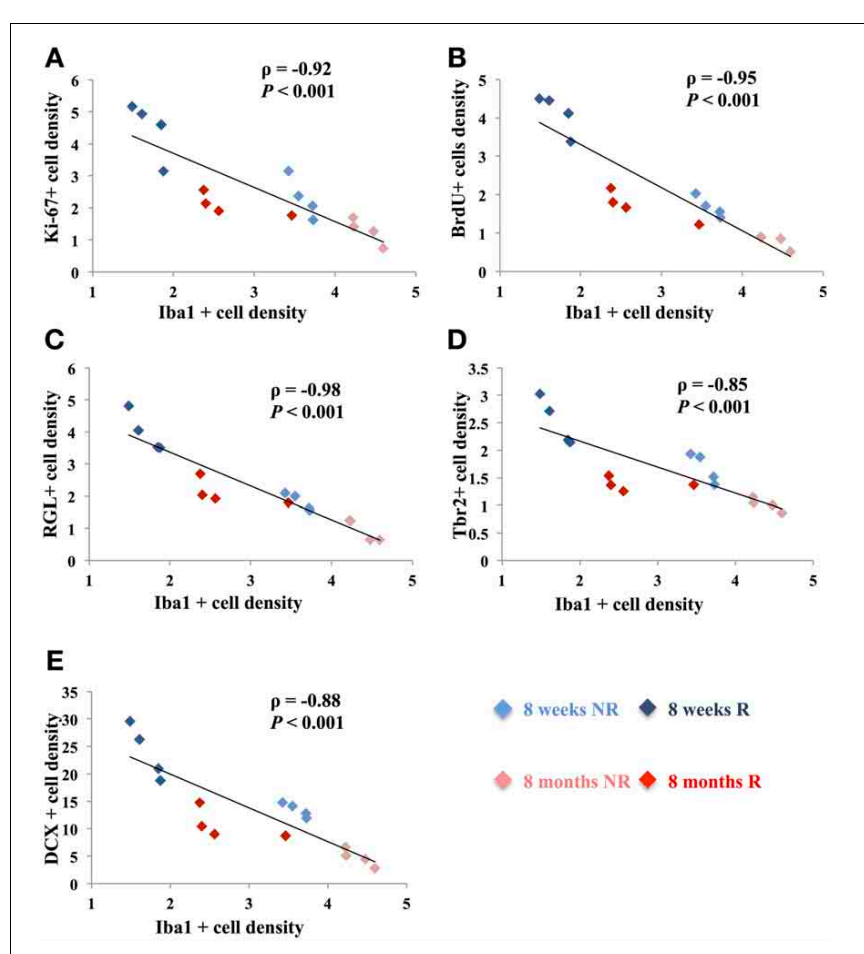

FIGURE 3 | Correlation between cell proliferation and lba1-expressing microglia. (A-E) Plots showing the density of Ki-67-expressing cells (A), BrdU-labeled cells (B), RGL cells (C), Tbr2-expressing cells (D) and DCX-expressing cells (E), as a function of the density of Iba1-expressing cells, in the dentate gyrus. Each point represents the density of cells for one animal. Spearman's correlation test was used to study the correlation, $\rho$ : coefficient of Spearman; $p$ : value of significance.

et al., 2007; Kohman et al., 2012). Similarly, aging is associated with a reduced neurogenesis, mild, chronic inflammation and increased microglia proliferation that can be attenuated by voluntary running (Kohman et al., 2012). In these experimental paradigms, inflammatory microglia is believed to directly inhibit neurogenesis, since anti-inflammatory treatments restore neurogenesis (Ekdahl et al., 2003; Monje et al., 2003; Liu et al., 2007; Kohman and Rhodes, 2013) and the exercise-induced increase and the age-dependent decline in neurogenesis are mediated by microglia in vitro (Vukovic et al., 2012).

However, in our study, we did not induce inflammation and in absence of activation, microglia has been reported to promote neurogenesis: In adult rats, the increased neurogenesis induced by environmental enrichment was accompanied by an increase in microglia number, whereas immunodeficient mice had impaired neurogenesis (Ziv et al., 2006). Similarly, voluntary running was shown to increase neurogenesis (van Praag et al., 1999) and microglia proliferation (Ehninger and Kempermann, 2008; Encinas et al., 2011), without inducing changes in the total number of microglia cells or in their activation state (Encinas et al., 2011). In vitro too, microglia was shown to promote the proliferation of co-cultured NPCs, an effect believed to be mediated by released factors, since the pro-neurogenic effect was mimicked by microglia-conditioned medium (Morgan et al., 2004; Aarum et al., 2003; Walton et al., 2006; Nakanishi et al.,
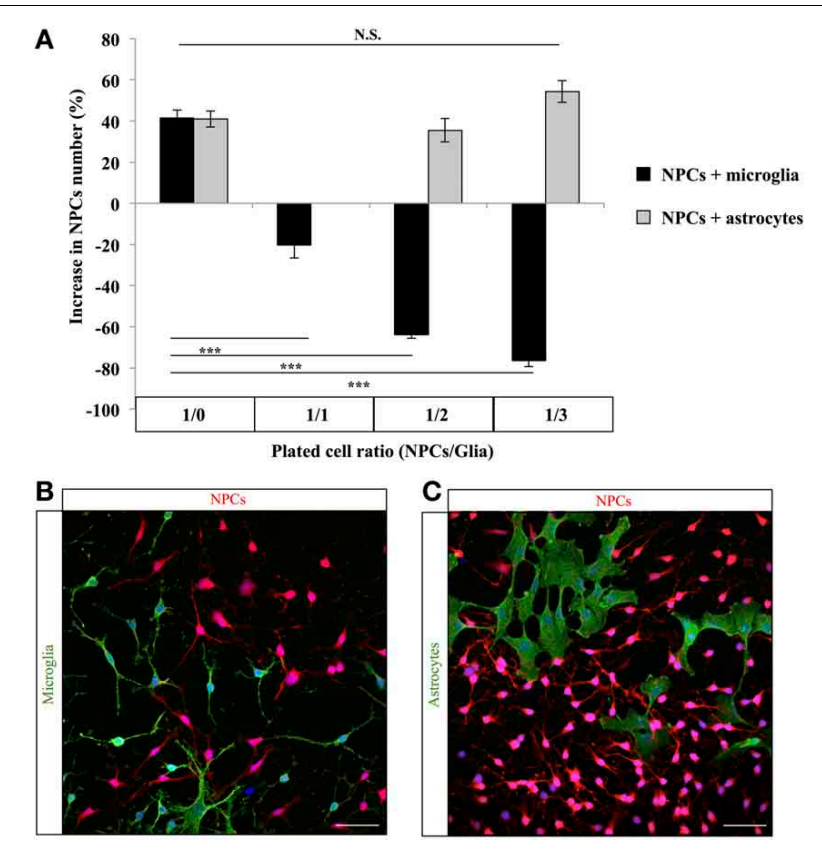

FIGURE 4 | Effect of microglia on progenitor cells in vitro. (A) Histogram of the change in NPCs number when cultured with microglia or astrocytes ( $\%$ from the number of plated cells). $N=3$ culture wells per group. (B) Confocal maximal projection micrographs of NPCs-microglia co-cultures. (C) Confocal maximal projection micrographs of NPCs-astrocytes co-cultures. N.S.: $p>0.05,{ }^{* * *} p<0.001$. Each value represents the mean \pm SEM.

2007). Thus, it is surprising that in our study, we observed an inverse correlation between microglia number and neurogenesis, in absence of inflammatory stimulus, in particular in running and non-running young mice. This discrepancy may partly result from differences in housing conditions, diet or animal strain. Indeed, while our study examined transgenic mice in the FVB/N background, the Ziv study used adult male rats (Ziv et al., 2006) and the Olah study used adult male $\mathrm{C} 57 \mathrm{Bl} / 6$ mice (Olah et al., 2009). FVB/N mice are known to have reduced cell proliferation in the adult DG, as compared to C57Bl/6 mice (Schauwecker, 2006) and may therefore respond differently to microglia regulation, or have increased basal inflammatory state. However, the absence of inflammatory stimulus in our in vivo experiments as well as the morphological aspect of microglia suggestive of a resting state, indicate no overt inflammation in GFAP-GFP mice. Furthermore, the observation that microglia reduced the number of NPCs in vitro supports the idea of a direct inhibition of progenitor cell proliferation by microglia. Finally, in a recent study, we found that doxycycline treatment decreased microglia cell number and increased neurogenesis and cell proliferation in C57Bl/6 mice (Sultan et al., 2013), suggesting that environmental factors and housing conditions may interfere with the effect of microglia on adult neurogenesis, rather than mouse strain.

However, correlation does not imply causality and the signaling between microglia and neurogenesis in the adult healthy brain remains unclear. Although activated microglia can directly inhibit neurogenesis by releasing a number of pro-inflammatory 
cytokines (Monje et al., 2003; Nakanishi et al., 2007), external factors such as voluntary running may act on neurogenesis independently from microglia. For example, the exercise-induced increase in neurogenesis depends on peripheral VEGF (Fabel et al., 2003) that acts directly on NPCs of the hippocampus (Fournier et al., 2012). Running can also affect microglia by reducing the expression of pro-inflammatory cytokines such as TNF- $\alpha$, and increasing the expression of the anti-inflammatory cytokines such as IL-1ra (Pervaiz and Hoffman-Goetz, 2011) or the chemokine $\mathrm{CX}_{3} \mathrm{CL} 1$, that induces a neuroprotective microglia phenotype and promotes neurogenesis (Vukovic et al., 2012). Inversely, gene expression analysis in the hippocampus showed that running induced the transcription of genes involved in inflammation, including genes related to MHC I ( $\beta 2-$ microglobulin, H2-D1) and elements of the complement system (C4A, C3, C1q) or in the inflammatory response (COX-2, CX3C), suggesting that running may increase inflammation (Tong et al., 2001; Kohman et al., 2011).

Depending on its activation state, microglia may have opposite effects on adult neurogenesis and it is likely that in the same brain, pro-neurogenic and anti-neurogenic microglia co-exist, with a different response to external stimulus, such as voluntary running and housing conditions. The combined action of external factors and microglia state may then result in unexpected effects

\section{REFERENCES}

Aarum, J., Sandberg, K., Haeberlein, S. L., and Persson, M. A. (2003). Migration and differentiation of neural precursor cells can be directed by microglia. Proc. Natl. Acad. Sci. U.S.A. 100, 15983-15988. doi: 10.1073/pnas. 2237050100

Aimone, J. B., Deng, W., and Gage, F. H. (2010). Adult neurogenesis: integrating theories and separating functions. Trends Cogn. Sci. 14, 325-337. doi: 10.1016/j.tics.2010. 04.003

Altman, J. (1969). Autoradiographic and histological studies of postnatal neurogenesis. 3. Dating the time of production and onset of differentiation of cerebellar microneurons in rats. J. Comp. Neurol. 136, 269-293. doi: 10.1002/cne. 901360303

Beckervordersandforth, R., Tripathi, P., Ninkovic, J., Bayam, E., Lepier, A., Stempfhuber, B., et al. (2010). In vivo fate mapping and expression analysis reveals molecular hallmarks of prospectively isolated adult neural stem cells. Cell Stem Cell 7, 744-758. doi: 10.1016/j.stem.2010. 11.017

Bonaguidi, M. A., Wheeler, M. A., Shapiro, J. S., Stadel, R. P., Sun, G. J., Ming, G. L., et al. (2011). In vivo clonal analysis reveals self-renewing and multipotent adult neural stem cell characteristics. Cell 145, 1142-1155. doi: 10.1016/j.cell.2011.05.024

Ehninger, D., and Kempermann, G. (2008). Neurogenesis in the adult hippocampus. Cell Tissue Res. 331, 0478-3

Ekdahl, C. T., Claasen, J. H., Bonde, S., Kokaia, Z., and Lindvall, O. (2003). Inflammation is detrimental for neurogenesis in adult brain. Proc. Natl. Acad. Sci. U.S.A. 100, 13632-13637. doi: 10.1073/ pnas. 2234031100

Encinas, J. M., Michurina, T. V., Peunova, N., Park, J. H., Tordo, J., Peterson, D. A., et al. (2011). Division-coupled astrocytic differentiation and age-related depletion of neural stem cells in the adult hippocampus. Cell Stem Cell 8, 566-579. doi: 10.1016/j.stem.2011.03.010

Eriksson, P. S., Perfilieva, E., BjorkEriksson, T., Alborn, A. M., Nordborg, C., Peterson, D. A., et al. (1998). Neurogenesis in the adult human hippocampus. Nat. Med. 4, 1313-1317. doi: 10.1038/ 3305

Fabel, K., Fabel, K., Tam, B., Kaufer, D., Baiker, A., Simmons, N., et al. (2003). VEGF is necessary for exercise-induced adult hippocampal neurogenesis. Eur. J. Neurosci. 18, 2803-2812. doi: 10.1111/j.1460-9568.2003.03041.x 243-250. doi: 10.1007/s00441-007-

on adult neurogenesis. Clearly, further experiments will be necessary to elucidate the regulation of neurogenesis by microglia and the role played by environmental factors such as stress and activity on both microglia and neurogenesis. This knowledge is crucial for the understanding of the regulation of adult neurogenesis by the neurogenic niche as well as for the therapeutic use of neural stem cells in inflammatory context such as lesions or neurodegenerative disorders.

\section{AUTHOR CONTRIBUTIONS}

Conceived and designed the experiments: Elias Gebara, Sebastien Sultan and Nicolas Toni. Performed the experiments: Elias Gebara, Sebastien Sultan and Jacqueline Kocher-Braissant Analyzed the data: Elias Gebara and Sebastien Sultan Wrote the paper: Elias Gebara, Sebastien Sultan and Nicolas Toni.

\section{ACKNOWLEDGMENTS}

The authors wish to thank Romain Gosselin (Department of Fundamental Neurosciences, University of Lausanne, Switzerland) for critical insight on in vitro experiments. Images acquisition was performed at the Cellular Imaging Facility of the University of Lausanne (Lausanne, Switzerland). This work was supported by the Swiss National Science Foundation and the Leenaards Foundation.

Fournier, N. M., Lee, B., Banasr, M., Elsayed, M., and Duman, R. S. (2012). Vascular endothelial growth factor regulates adult hippocampal cell proliferation through MEK/ERKand PI3K/Akt-dependent signaling. Neuropharmacology 63, 642-652. doi: 10.1016/ j.neuropharm.2012.04.033

Fujioka, H., and Akema, T. (2010). Lipopolysaccharide acutely inhibits proliferation of neural precursor cells in the dentate gyrus in adult rats. Brain Res. 1352, 35-42. doi 10.1016/j.brainres.2010.07.032

Gage, F. H. (2000). Mammalian Neural Stem Cells. Science 287, 1433-1438. doi: 10.1126/science.287.5457.1433

Gao, X., and Chen, J. (2013). Moderate traumatic brain injury promotes neural precursor proliferation without increasing neurogenesis in the adult hippocampus. Exp. Neurol. 239, 38-48. doi: 10.1016/j.expneurol.2012.09.012

Hodge, R. D., Kowalczyk, T. D., Wolf, S. A., Encinas, J. M., Rippey, C., Enikolopov, G., et al. (2008). Intermediate progenitors in adult hippocampal neurogenesis: Tbr2 expression and coordinate regulation of neuronal output. J. Neurosci. 28, 3707-3717. doi: 10.1523/JNEUROSCI.4280-07.2008 Huttmann, K., Sadgrove, M., Wallraff, A., Hinterkeuser, S., Kirchhoff,
F., Steinhauser, C., et al. (2003). Seizures preferentially stimulate proliferation of radial glia-like astrocytes in the adult dentate gyrus: functional and immunocytochemical analysis. Eur. J. Neurosci. 18, 2769-2778. doi: $\quad 10.1111 / j .1460-9568.2003$ 03002.x

Kempermann, G. (2011). Seven principles in the regulation of adult neurogenesis. Eur. J. Neurosci. 33, 1018-1024. doi: 10.1111/j.1460-9568.2011.07599.x

Keohane, A., Ryan, S., Maloney, E., Sullivan, A. M., and Nolan, Y. M. (2010). Tumour necrosis factor-alpha impairs neuronal differentiation but not proliferation of hippocampal neural precursor cells: role of hes1. Mol. Cell. Neurosci. 43, 127-135. doi: 10.1016/j.mcn.2009.10.003

Kohman, R. A., Deyoung, E. K., Bhattacharya, T. K., Peterson, L. N., and Rhodes, J. S. (2012). Wheel running attenuates microglia proliferation and increases expression of a proneurogenic phenotype in the hippocampus of aged mice. Brain Behav. Immun. 26, 803-810. doi: 10.1016/j.bbi.2011.10.006

Kohman, R. A., and Rhodes, J. S. (2013). Neurogenesis, inflammation and behavior. Brain Behav. Immun. 27, 22-32. doi: 10.1016/j.bbi.2012.09.003 
Kohman, R. A., Rodriguez-Zas, S. L., Southey, B. R., Kelley, K. W., Dantzer, R., and Rhodes, J. S. (2011). Voluntary wheel running reverses age-induced changes in hippocampal gene expression.PLoS ONE 6:e22654. doi: 10.1371/journal.pone.0022654

Kriegstein, A., and Alvarez-Buylla, A. (2009). The glial nature of embryonic and adult neural stem cells. Annu. Rev. Neurosci. 32, 149-184. doi: 10.1146/annurev.neuro.051508. 135600

Kuhn, H. G., Dickinson-Anson, H., and Gage, F. H. (1996). Neurogenesis in the dentate gyrus of the adult rat: age-related decrease of neuronal progenitor proliferation. J. Neurosci. 16, 2027-2033.

Liu, Z., Fan, Y., Won, S. J., Neumann, M., Hu, D., Zhou, L., et al. (2007). Chronic treatment with minocycline preserves adult new neurons and reduces functional impairment after focal cerebral ischemia. Stroke 38, 146-152. doi: 10.1161/01.STR. 0000251791.64910.cd

Mandyam, C. D., Harburg, G. C., and Eisch, A. J. (2007). Determination of key aspects of precursor cell proliferation, cell cycle length and kinetics in the adult mouse subgranular zone. Neuroscience 146, 108-122. doi: 10.1016/j.neuroscience.2006.12.064

Mignone, J. L., Kukekov, V., Chiang, A. S., Steindler, D., and Enikolopov, G. (2004). Neural stem and progenitor cells in nestin-GFP transgenic mice. J. Comp. Neurol. 469, 311-324. doi: 10.1002/cne.10964

Monje, M. L., Mizumatsu, S., Fike, J. R., and Palmer, T. D. (2002). Irradiation induces neural precursor-cell dysfunction. Nat. Med. 8, 955-962. doi: $10.1038 / \mathrm{nm} 749$

Monje, M. L., Toda, H., and Palmer, T. D. (2003). Inflammatory blockade restores adult hippocampal neurogenesis. Science 302, 1760-1765. doi: 10.1126/science. 1088417

Morgan, S. C., Taylor, D. L., and Pocock, J. M. (2004). Microglia release activators of neuronal proliferation mediated by activation of mitogen-activated protein kinase, phosphatidylinositol-3-kinase/Akt and delta-Notch signalling cascades. J. Neurochem. 90, 89-101. doi: 10.1111/j.1471-4159.2004.02461.x

Mosher, K. I., Andres, R. H., Fukuhara, T., Bieri, G., Hasegawa-Moriyama,
M., He, Y., et al. (2012). Neural progenitor cells regulate microglia functions and activity. Nat. Neurosci. 15, 1485-1487. doi: 10.1038/nn.3233

Nakanishi, M., Niidome, T., Matsuda, S., Akaike, A., Kihara, T., and Sugimoto, H. (2007). Microgliaderived interleukin- 6 and leukaemia inhibitory factor promote astrocytic differentiation of neural stem/progenitor cells. Eur. J. Neurosci. 25, 649-658. doi: 10.1111/j.1460-9568.2007.05309.x

Nolte, C., Matyash, M., Pivneva, T., Schipke, C. G., Ohlemeyer, C., Hanisch, U. K., et al. (2001). GFAP promoter-controlled EGFPexpressing transgenic mice: a tool to visualize astrocytes and astrogliosis in living brain tissue. Glia 33, 72-86.

Olah, M., Ping, G., De Haas, A. H., Brouwer, N., Meerlo, P., Van Der Zee, E. A., et al. (2009). Enhanced hippocampal neurogenesis in the absence of microglia $\mathrm{T}$ cell interaction and microglia activation in the murine running wheel model. Glia 57, 1046-1061. doi: 10.1002/glia.20828

Palmer, T. D., Takahashi, J., and Gage, F. H. (1997). The adult rat hippocampus contains primordial neural stem cells. Mol. Cell Neurosci. 8, 389-404. doi: 10.1006/mcne.1996.0595

Paolicelli, R. C., Bolasco, G., Pagani, F., Maggi, L., Scianni, M., Panzanelli, P., et al. (2011). Synaptic pruning by microglia is necessary for normal brain development. Science 333, 1456-1458. doi: $10.1126 /$ science. 1202529

Pervaiz, N., and Hoffman-Goetz, L. (2011). Freewheel training alters mouse hippocampal cytokines. Int J. Sports Med. 32, 889-895. doi: 10.1055/s-0031-1279780

Schauwecker, P. E. (2006). Genetic influence on neurogenesis in the dentate gyrus of two strains of adult mice. Brain Res. 1120, 83-92. doi: 10.1016/j.brainres.2006.08.086

Shihabuddin, L. S., Horner, P. J., Ray, J., and Gage, F. H. (2000). Adult spinal cord stem cells generate neurons after transplantation in the adult dentate gyrus. J. Neurosci. 20, 8727-8735.

Sierra, A., Encinas, J. M., Deudero, J. J., Chancey, J. H., Enikolopov, G., Overstreet-Wadiche, L. S., et al. (2010). Microglia shape adult hippocampal neurogenesis through apoptosis-coupled phagocytosis.
Cell Stem Cell 7, 483-495. doi: 10.1016/j.stem.2010.08.014

Song, H., Stevens, C. F., and Gage, F. H. (2002). Astroglia induce neurogenesis from adult neural stem cells. Nature 417, 39-44. doi: $10.1038 / 417039 \mathrm{a}$

Sultan, S., Gebara, E., and Toni, N. (2013). Doxycycline increases neurogenesis and reduces microglia in the adult hippocampus. Front. Neurosci. 7:131. doi: 10.3389/fnins.2013.00131

Taupin, P. (2007). BrdU immunohistochemistry for studying adult neurogenesis: paradigms, pitfalls, limitations, and validation. Brain Res. Rev. 53, 198-214. doi: 10.1016/j.brainresrev.2006.08.002

Thuret, S., Toni, N., Aigner, S., Yeo, G. W., and Gage, F. H. (2009). Hippocampus-dependent learning is associated with adult neurogenesis in MRL/MpJ mice. Hippocampus 19, 658-669. doi: 10.1002/hipo. 20550

Tong, L., Shen, H., Perreau, V M., Balazs, R., and Cotman, C. W. (2001). Effects of exercise on gene-expression profile in the rat hippocampus. Neurobiol. Dis. 8, 1046-1056. doi: 10.1006/nbdi.2001.0427

Toni, N., and Sultan, S. (2011). Synapse formation on adult-born hippocampal neurons. Eur. J. Neurosci. 33, 1062-1068. doi: 10.1111/j.1460-9568.2011.07604.x

Vallieres, L., Campbell, I. L., Gage, F. H., and Sawchenko, P. E. (2002) Reduced hippocampal neurogenesis in adult transgenic mice with chronic astrocytic production of interleukin-6. J. Neurosci. 22, 486-492.

van Praag, H., Kempermann, G., and Gage, F. H. (1999). Running increases cell proliferation and neurogenesis in the adult mouse dentate gyrus. Nat. Neurosci. 2, 266-270. doi: 10.1038/6368

van Praag, H., Shubert, T., Zhao, C., and Gage, F. H. (2005). Exercise enhances learning and hippocampal neurogenesis in aged mice. J. Neurosci. 25, 8680-8685. doi 10.1523/JNEUROSCI.1731-05.2005

Vukovic, J., Colditz, M. J., Blackmore, D. G., Ruitenberg, M. J., and Bartlett, P. F. (2012). Microglia modulate hippocampal neural precursor activity in response to exercise and aging. J. Neurosci. 32, 6435-6443.
Walton, N. M., Sutter, B. M., Laywell, E. D., Levkoff, L. H., Kearns, S. M., Marshall, G. P., et al. (2006). Microglia instruct subventricular zone neurogenesis. Glia 54, 815-825. doi: 10.1002/glia.20419

Yang, C. P., Gilley, J. A., Zhang, G., and Kernie, S. G. (2011). ApoE is required for maintenance of the dentate gyrus neural progenitor pool. Development 138, 4351-4362. doi: $10.1242 /$ dev.065540

Yao, J., Mu, Y., and Gage, F. H. (2012). Neural stem cells: mechanisms and modeling. Protein Cell 3, 251-261.

Zhao, C., Deng, W., and Gage, F. H. (2008). Mechanisms and functional implications of adult neurogenesis. Cell 132, 645-660. doi: 10.1016/j.cell.2008.01.033

Ziv, Y., Ron, N., Butovsky, O., Landa, G., Sudai, E., Greenberg, N., et al. (2006). Immune cells contribute to the maintenance of neurogenesis and spatial learning abilities in adulthood. Nat. Neurosci. 9, 268-275. doi: 10.1038/nn1629

Conflict of Interest Statement: The authors declare that the research was conducted in the absence of any commercial or financial relationships that could be construed as a potential conflict of interest.

Received: 23 June 2013; paper pending published: 13 July 2013; accepted: 30 July 2013; published online: 20 August 2013. Citation: Gebara E, Sultan S, KocherBraissant J and Toni N (2013) Adult hippocampal neurogenesis inversely correlates with microglia in conditions of voluntary running and aging. Front. Neurosci. 7:145. doi: 10.3389/fnins. 2013.00145

This article was submitted to Neurogenesis, a section of the journal Frontiers in Neuroscience.

Copyright (c) 2013 Gebara, Sultan, Kocher-Braissant and Toni. This is an open-access article distributed under the terms of the Creative Commons Attribution License (CC BY). The use, distribution or reproduction in other forums is permitted, provided the original author(s) or licensor are credited and that the original publication in this journal is cited, in accordance with accepted academic practice. No use, distribution or reproduction is permitted which does not comply with these terms. 